\title{
Group graded basic Morita equivalences and the Harris-Knörr correspondence
}

\author{
Andrei Marcus \\ Communicated by Britta Späth
}

\begin{abstract}
Let $G$ be a finite group, let $b$ be a $G$-invariant block with defect group $Q$ of the normal subgroup $H$ of $G$, and let $b^{\prime} \in Z\left(\mathcal{O} N_{H}(Q)\right)$ be the Brauer correspondent of $b$. We show that the bijection between the blocks of $G$ covering $b$ and the blocks of $N_{G}(Q)$ covering $b^{\prime}$, induced by a $G / H$-graded basic Morita equivalence between the block extensions $b \mathcal{O} G$ and $b^{\prime} \mathcal{O} N_{G}(Q)$, coincides with the Harris-Knörr correspondence.
\end{abstract}

\section{Introduction}

The Clifford theoretical approach to Broué's abelian defect group conjecture involves group graded categorical equivalences between block extensions, which occur by taking a normal subgroup of the finite group $G$, and a $\bar{G}$-invariant block $b$ of the group algebra $\mathcal{O} H$, where $\bar{G}=G / H$ and $\mathcal{O}$ is a complete discrete valuation ring. Let $Q \leq H$ be a defect group of $b$, and let $b^{\prime}$ be the Brauer correspondent in $\mathcal{O} H^{\prime}$, where $H^{\prime}=N_{H}(Q)$. A very useful situation is when an equivalence between the block algebras $b \mathcal{O} H$ and $b^{\prime} \mathcal{O} H^{\prime}$ can be lifted to an equivalence between the block extensions $b \mathcal{O} G$ and $b^{\prime} \mathcal{O} G^{\prime}$, where $G^{\prime}=N_{G}(Q)$, induced by (complexes of) $\bar{G}$-graded bimodules. Then such a $\bar{G}$-graded equivalence induces a bijection between the blocks of $b \mathcal{O} G$ and those of $b^{\prime} \mathcal{O} G^{\prime}$.

On the other hand, a well-known result of M. E. Harris and R. Knörr [11] states that the Brauer correspondence induces a bijection between the blocks of $\mathcal{O} G$ covering $b$ and the blocks of $\mathcal{O} N_{G}(Q)$ covering $b^{\prime}$. It is natural to ask what the relationship is between these two correspondences.

Another motivation comes from the work of Britta Späth on reduction methods for local-global conjectures, presented in the surveys [19,20]. One of the important tools is the relation $\geq_{b}$ between character triples (see [19, Definition 3.2] and [20, Definition 4.2]), which also involves the Harris-Knörr correspondence.

We prove in Corollary 4.4 that the bijection induced by a $\bar{G}$-graded Morita equivalence between $b \mathcal{O} G$ and $b^{\prime} \mathcal{O} G^{\prime}$ (see [4-7]) coincides with the Harris-Knörr correspondence. 
To this end, we use the stabilizer $\bar{G}[b]$ of $b \mathcal{O} H$ regarded as a bimodule, and the Clifford extension of the block $b$, introduced by E. C. Dade in $[8,9]$. Recall that the Clifford extension of $b$, denoted here by $\bar{C}$, is the centralizer $C_{b \mathcal{O} G}(b \mathcal{O} H)$ modulo its $\bar{G}$-graded Jacobson radical. This is invariant under $\bar{G}$-graded Morita equivalence, and Dade [9] gives a bijective correspondence between the blocks of $G$ covering $b$ and the $\bar{G}$-conjugacy classes of blocks of $\bar{C}$, and he also proves that the blocks $b$ and $b^{\prime}$ have isomorphic Clifford extensions $\bar{C}$ and $\bar{C}^{\prime}$, this isomorphism being induced by the Brauer map.

In Section 2, we introduce our notation, and we recall the necessary facts on basic Morita equivalences. In Section 3, we discuss the Harris-Knörr correspondence in terms of isomorphism of the Clifford extensions of the blocks $b$ and $b^{\prime}$. Finally, our main result, Corollary 4.4, is a consequence of Theorem 4.3, which says that the isomorphism $\bar{C} \simeq \bar{C}^{\prime}$ induced by the Brauer map is invariant under $\bar{G}$-graded basic Morita equivalences.

These results can be generalized to $\bar{G}$-graded Rickard equivalences. We intend to deal with this aspect in a subsequent paper, as well as with the precise relationship with Späth's relation $\geq_{b}$ and with Turull's approach [21] to character triple isomorphisms.

\section{Preliminaries and quoted results}

Notation 2.1. Let $\mathcal{O}$ be a complete discrete valuation ring with residue field $k$ of characteristic $p>0$. For the sake of simplicity, we assume that $k$ is algebraically closed.

Let $H$ be a normal subgroup of the finite group $G$, and let $b$ be a $G$-invariant block of $\mathcal{O} N$, that is, $\{b\}$ is a point of $G$ on $\mathcal{O} N$. We denote

$$
\bar{G}:=G / H, \quad A:=\mathcal{O} G b, \quad B:=\mathcal{O} H b,
$$

and we regard $A$ as a $\bar{G}$-graded $\mathcal{O}$-algebra with identity component $B$.

2.2. Take a defect pointed group $P_{\gamma}$ of $G_{\{b\}}$. Then, by [12, Proposition 5.3], there is a defect pointed group $Q_{\delta}$ of $H_{\{b\}}$ on the $H$-interior algebra $\mathcal{O} H$ such that $Q_{\delta} \leq P_{\gamma}$; in this case, we may assume that $Q=P \cap H$.

The Frattini argument implies that $G=H N_{G}\left(Q_{\delta}\right)$; hence

$$
\bar{G} \simeq N_{G}(Q) / N_{H}(Q) \simeq N_{G}\left(Q_{\delta}\right) / N_{H}\left(Q_{\delta}\right) .
$$

Let $i \in \gamma$ and $j \in \delta$ be source idempotents such that $j=i j=j i$. Set

$$
A_{\gamma}=i A i, \quad A_{\delta}=j A j, \quad B_{\delta}=j B j, \quad B_{\gamma}=i B i .
$$


By [15, Proposition 3.2], both $A_{\delta}$ and $A_{\gamma}$ are strongly $\bar{G}$-graded $\mathcal{O}$-algebras, and we have $\bar{G}$-graded Morita equivalences between $A, A_{\delta}$ and $A_{\gamma}$.

2.3. Recall (see $[13, \S 5.9])$ that there is a maximal $(H, b)$-Brauer pair, denoted $\left(Q, b_{\delta}\right)$, associated with $Q_{\delta}$. Here $b_{\delta}$ is a block of ${ }_{k} C_{H}(Q)$ with defect group $Z(Q)$. It is well known that $b_{\delta}$ lifts uniquely to a block (still denoted by $b_{\delta}$ ) of $\mathcal{O} C_{H}(Q)$ with defect group $Z(Q)$, and that we have the equality

$$
N_{H}\left(Q_{\delta}\right)=N_{H}\left(Q, b_{\delta}\right) .
$$

Moreover, $b_{\delta}$ is also a block of $\mathcal{O} Q C_{H}(Q)$ with defect group $Q$. We denote

$$
\mathcal{A}:=\mathcal{O} N_{G}\left(Q_{\delta}\right) b_{\delta}, \quad \mathcal{B}:=\mathcal{O} N_{H}\left(Q_{\delta}\right) b_{\delta},
$$

and we will regard $\mathcal{A}$ as a $\bar{G}$-graded algebra with 1 -component $\mathscr{B}$.

Notation 2.4. Consider another finite group $G^{\prime}$, and assume that $\omega: G \rightarrow \bar{G}$ and $\omega^{\prime}: G^{\prime} \rightarrow \bar{G}$ are group epimorphisms with $H=\operatorname{Ker} \omega$ and $H^{\prime}=\operatorname{Ker} \omega^{\prime}$. Let $b^{\prime}$ be a $G^{\prime}$-invariant block of $\mathcal{O} H^{\prime}$, and let

$$
A^{\prime}:=\mathcal{O} G^{\prime} b^{\prime}, \quad B^{\prime}:=\mathcal{O} H^{\prime} b^{\prime},
$$

$A^{\prime}$ as a $\bar{G}$-graded $G^{\prime}$-interior algebra with 1 -component $B^{\prime}$. We will use ${ }^{\prime}$ to denote the objects associated with $b^{\prime}$.

Set $\ddot{G}:=\left(\omega \times \omega^{\prime}\right)^{-1}(\Delta(\bar{G}))$ and $\Delta:=\left(b \otimes b^{\prime}\right) \mathcal{O} \ddot{G}$. In particular, any $\Delta$-module, is, by restriction of scalars, a $\left(B, B^{\prime}\right)$-bimodule.

2.5. Basic Morita equivalences were introduced by Puig [16]; their local properties have been further studied in [18], and these results have been generalized to block extensions in [4-7]. According to [5, Definition 4.2], we say that $A$ is $\bar{G}$-graded basic Morita equivalent to $A^{\prime}$ if there is an indecomposable $\Delta$-module $M$ with vertex $\ddot{P} \leq \ddot{G}$ and endopermutation source $\ddot{N}$ such that there is an embedding

$$
f: A_{\gamma} \rightarrow S \otimes A_{\gamma^{\prime}}^{\prime}
$$

of $\bar{G}$-graded $P$-interior algebras, where $S:=\operatorname{End}_{\mathcal{O}}(\ddot{N})$ is a Dade $\ddot{P}$-algebra. Recall that, in this situation, by [5, Proposition 4.1], the projections from $G \times G^{\prime}$ to $G$ and $G^{\prime}$ induce isomorphisms $\ddot{P} \simeq P \simeq P^{\prime}$, so we can again identify the vertex $\ddot{P}$ with the defect groups $P$ and $P^{\prime}$, and this identification gives the $P$-interior algebra structure on $S \otimes A_{\gamma^{\prime}}^{\prime}$.

Note also that, by [5, Corollary 4.3], $B$ and $B^{\prime}$ are basic Morita equivalent because the embedding $f$ restricts to a $Q$-interior $P$-algebra embedding

$$
f: B_{\gamma} \rightarrow S \otimes B_{\gamma^{\prime}}^{\prime}
$$


Now let $R \leq P$ and $R^{\prime} \leq P^{\prime}$ such that $R^{\prime}$ corresponds to $R$ via the identification of $\ddot{P}$ with $P$ and $P^{\prime}$. By applying the Brauer construction to (2.1), we obtain the $N_{P}(R)$-algebra embedding

$$
\bar{f}: B_{\gamma}(R) \rightarrow S(R) \otimes B_{\gamma^{\prime}}^{\prime}\left(R^{\prime}\right) .
$$

If $R_{\epsilon}$ is a local pointed subgroup of $P_{\gamma}$, then, by (2.2), there is a unique local pointed group $R_{\epsilon^{\prime}}^{\prime}$ such that $R_{\epsilon^{\prime}}^{\prime} \leq P_{\gamma^{\prime}}^{\prime}$. We obtain in this way a bijection $R_{\epsilon} \leftrightarrow R_{\epsilon^{\prime}}^{\prime}$ between the local pointed groups included in $P_{\gamma}$ and local pointed groups included in $P_{\gamma^{\prime}}^{\prime}$. We will use this bijection in 2.6 and in Section 4, in the particular case when $R_{\epsilon}=Q_{\delta}$.

2.6. Finally, recall also that the $\bar{G}$-graded basic Morita equivalence between $A$ and $A^{\prime}$ induces a $\bar{G}$-graded basic Morita equivalence between $\mathcal{A}=\mathcal{O} N_{G}\left(Q_{\delta}\right) b_{\delta}$ and $\mathcal{A}^{\prime}=\mathcal{O} N_{G^{\prime}}\left(Q_{\delta^{\prime}}\right) b_{\delta^{\prime}}^{\prime}$, which is obtained as follows (see [5, Lemma 6.6 and 6.7] and [7, Proof of Theorem 1.1]).

Let $\lambda=\operatorname{br}_{Q}(\gamma)$ and $\lambda^{\prime}=\operatorname{br}_{Q}\left(\gamma^{\prime}\right)$. Then $P_{\lambda}$ is a defect pointed group of $b_{\delta}$ in $N_{G}\left(Q_{\delta}\right)$, and $P_{\lambda^{\prime}}$ is a defect pointed group of $b_{\delta^{\prime}}^{\prime}$ in $N_{G^{\prime}}\left(Q_{\delta^{\prime}}\right)$. It follows that $\mathcal{A}_{\lambda}$ is the source algebra of $\mathcal{A}$, and $\mathcal{A}_{\lambda^{\prime}}^{\prime}$ is the source algebra of $\mathcal{A}^{\prime}$.

Note that $S(Q)$ is a Dade $P$-interior algebra. By applying the extended Brauer construction [6, Section 4] to the embedding $f$, we get the embedding

$$
\mathcal{A}_{\lambda} \rightarrow S(Q) \otimes \mathcal{A}_{\lambda^{\prime}}^{\prime}
$$

of $\bar{G}$-graded $P$-interior algebras, which gives the $\bar{G}$-graded basic Morita equivalence between $\mathcal{A}$ and $\mathcal{A}^{\prime}$.

\section{Covering blocks and Clifford extensions}

3.1. Let $A=b \mathcal{O} G, B=b \mathcal{O} H, \mathcal{A}=\mathcal{O} N_{G}\left(Q_{\delta}\right) b_{\delta}$ and $\mathscr{B}=\mathcal{O} N_{H}\left(Q_{\delta}\right) b_{\delta}$ as in Section 2, and let $A^{\prime}=b^{\prime} \mathcal{O} N_{G}(Q), B^{\prime}=b^{\prime} \mathcal{O} N_{H}(Q)$, where $\left.b^{\prime}=\operatorname{Tr}_{N_{H}}^{N_{H}(Q)} Q_{\delta}\right) b_{\delta}$. The Fong-Reynolds equivalence applied in this situation says that there is a $\bar{G}$ graded basic Morita equivalence between $A^{\prime}$ and $\mathcal{A}$.

3.2. Consider the centralizer in $A$ of the 1-component $B$,

$$
C:=C_{A}(B)=(b \mathcal{O} G)^{H} .
$$

We know that $C$ is a $\bar{G}$-graded $\bar{G}$-algebra, which is not strongly graded in general. To obtain a crossed product, we consider the stabilizer $\bar{G}[b]$ of $B$ as a $(B, B)$-bimodule, that is (see [9, Section 2.9]),

$$
\bar{G}[b]=\left\{\bar{g} \in \bar{G} \mid A_{\bar{g}} \simeq B \text { as }(B, B) \text {-bimodules }\right\}=\left\{\bar{g} \in \bar{G} \mid A_{\bar{g}} A_{\bar{g}}-1=B\right\} .
$$


Then $\bar{G}[b]$ is a normal subgroup of $\bar{G}$, and

$$
C_{\bar{G}[b]}=\bigoplus_{\bar{g} \in \bar{G}[b]} C_{\bar{g}}
$$

is a strongly $\bar{G}[b]$-graded $\bar{G}$-acted subalgebra of $C$. Regarding the graded Jacobson radical (see [9, Lemma 3.3], [14, Section 1.5.A]), we know that

$$
J_{\mathrm{gr}}(C)=\left(\bigoplus_{\bar{g} \in \bar{G} \backslash \bar{G}[b]} C_{\bar{g}}\right) \bigoplus\left(J_{\mathrm{gr}}(C[b])\right) ;
$$

hence

$$
\bar{C}=\bar{C}_{A}(B):=C / J_{\mathrm{gr}}(C) \simeq C_{\bar{G}[b]} / J_{\mathrm{gr}}\left(C_{\bar{G}[b]}\right)
$$

as $\bar{G}[b]$-graded $\bar{G}$-acted algebras.

Recall also that $J_{\text {gr }}(C) \subseteq J(C)$. It follows from this isomorphism that the primitive idempotents of $C$ lie in $C_{\bar{G}[b]}$; hence the $G$-invariant primitive idempotents of $C$ (that is, the blocks of $b \mathcal{O} G$ ) coincide with the $G$-invariant primitive idempotents of $C_{\bar{G}[b]}$.

3.3. In a similar way, we define the group $\bar{G}\left[b^{\prime}\right]$ and the $\bar{G}$-graded $\bar{G}$-acted algebras

$$
C^{\prime}:=C_{A^{\prime}}\left(B^{\prime}\right)=\left(b^{\prime} \mathcal{O} N_{G}(Q)\right)^{N_{H}(Q)} \quad \text { and } \quad \bar{C}^{\prime}=\bar{C}_{A^{\prime}}\left(B^{\prime}\right)=C^{\prime} / J_{\mathrm{gr}}\left(C^{\prime}\right),
$$

respectively the group $\bar{G}\left[b_{\delta}\right]$ and the $\bar{G}$-graded $\bar{G}$-algebras

$$
\varphi=C_{\mathcal{A}}(\mathscr{B}) \text { and } \bar{\varphi}=\bar{C}_{\mathscr{A}}(\mathscr{B})=\varphi / J_{\mathrm{gr}}(\varphi) \text {. }
$$

By [14, Corollary 5.1.4], the $\bar{G}$-graded Morita equivalence between $A^{\prime}$ and $\mathcal{A}$ induces the group isomorphism $\bar{G}\left[b^{\prime}\right] \simeq \bar{G}\left[b_{\delta}\right]$ and the following commutative diagram:

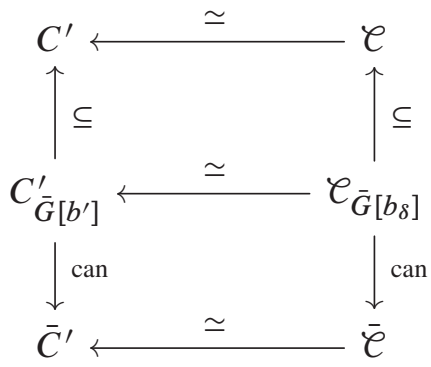

The main result of this section, which relates these algebras with the Brauer correspondence, is due to T. Coconeț [2], and originates from the work of E. C. Dade $[8,9]$. For convenience, we include here a complete proof which does not depend on the more general results from [3]. We need the following lemma. 
Lemma 3.4. Let $Q \leq D \leq P$. The Brauer homomorphism $\operatorname{br}_{Q}$ determines a bijection between the blocks of $\mathcal{O} G$ with defect group $D$ and the blocks of $\mathcal{O} N_{G}(Q)$ with defect group D. This correspondence coincides with the Brauer correspondence determined by br $_{D}$.

Proof. By [1, Proposition 1.5], applied to the appropriate restrictions of the Brauer map, we obtain the commutative diagram

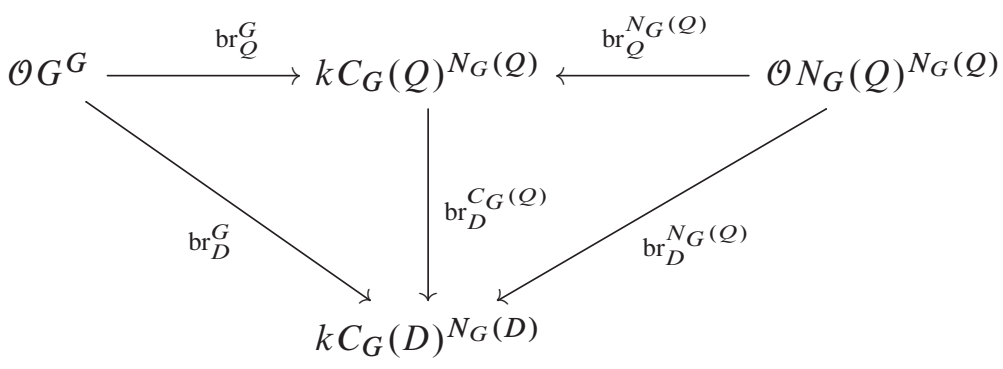

of homomorphisms of $N_{G}(Q)$-algebras.

Let $a$ be a block of $\mathcal{O} G$ with defect group $D$. Then, by the commutativity of the above diagram, $\tilde{a}:=\operatorname{br}_{Q}^{G}(a)$ is a primitive idempotent (hence block) of $k C_{G}(Q)^{N_{G}(Q)}$ with defect group $D$. It is easy to see that $\tilde{a}$ lifts to a block $a^{\prime}$ of $k N_{G}(Q)$, still with defect group $D$, that verifies $\operatorname{br}_{Q}^{N_{G}(Q)}\left(a^{\prime}\right)=\tilde{a}$. In this way, we obtain the bijection determined by br $_{Q}$.

Now let $a^{\prime \prime}$ denote the Brauer correspondent block of $a$ in $N_{G}(Q)$. This means that $a^{\prime \prime}$ belongs to $\mathcal{O} N_{G}(Q)$ and verifies $\operatorname{br}_{D}^{N_{G}(Q)}\left(a^{\prime \prime}\right)=\operatorname{br}_{D}^{G}(a)$. On the other hand, we get

$$
\operatorname{br}_{D}^{G}(a)=\operatorname{br}_{D}^{C_{G}(Q)}\left(\operatorname{br}_{Q}^{G}(a)\right)=\operatorname{br}_{D}^{C_{G}(Q)}\left(\operatorname{br}_{Q}^{N_{G}(Q)}(a)\right)=\operatorname{br}_{D}^{N_{G}(Q)}\left(a^{\prime}\right) .
$$

Finally, $a^{\prime}$ and $a^{\prime \prime}$ must coincide since, otherwise, we have $a^{\prime} \cdot a^{\prime \prime}=0$, which would imply $\operatorname{br}_{D}^{G}(a)=0$, a contradiction.

Remark 3.5. (a) Note that the statement of Lemma 3.4 is true under more general assumptions. All we need is that $D$ and $Q$ are $p$-subgroups of $G$ with $Q \unlhd D$ and $N_{G}(D) \leq N_{G}(Q)$.

(b) An immediate consequence of Lemma 3.4 is the main result of [11]: the Brauer map br $_{Q}$ determines a bijection between the blocks of $b \mathcal{O} G$ and those of $b^{\prime} \mathcal{O} N_{G}(Q)$, and this correspondence coincides with the Brauer correspondence between the covering blocks of $b$ and of $b^{\prime}$ respectively. Recall also that any block $a$ of $\mathcal{O} G$ covering $b$ has a defect group $D$ such that $Q \leq D \leq P$ and $D \cap H=Q$. 
Remark 3.6. The Brauer quotient of $C$ is

$$
C(Q)=\left((b \mathcal{O} G)^{H}\right)(Q)=(b \mathcal{O} G)(Q)^{N_{H}(Q)}=b^{\prime} k C_{G}(Q)^{N_{H}(Q)} .
$$

Note that, since $Q \subseteq H, C(Q)$ is a $\bar{G}$-graded $\bar{G}$-algebra. The image of the restriction of br $Q$ to $C_{\bar{G}[b]}$ is the strongly $\bar{G}[b]$-graded $\bar{G}$-subalgebra $\left(C_{\bar{G}[b]}\right)(Q)$ of $b^{\prime} k C_{G}(Q)^{N_{H}(Q)}$.

Theorem 3.7. The Brauer homomorphism br $_{Q}$ induces an isomorphism of $\bar{G}$ graded $\bar{G}$-acted algebras between $\bar{C}[b]$ and $\bar{C}^{\prime}$. Furthermore, this induces a bijection between the blocks of $G$ covering $b$ and the blocks of $N_{G}(Q)$ covering $b^{\prime}$, which coincides with the Harris-Knörr correspondence.

Proof. Note that $C^{\prime}=b^{\prime} \mathcal{O} C_{G}(Q)^{N_{H}(Q)} \oplus S$, where $S$ consists of $N_{H}(Q)$-fixed elements belonging to the radical of $b^{\prime} \mathcal{O} N_{G}(Q)$. It follows that $S=\bigoplus_{\bar{x} \in T} C_{\bar{x}}^{\prime}$ for some subset $T$ of $\bar{G} \backslash \bar{G}\left[b^{\prime}\right]$. We immediately deduce the equality

$$
C^{\prime}=b^{\prime} \mathcal{O} C_{G}(Q)^{N_{H}(Q)}+J_{\mathrm{gr}}\left(C^{\prime}\right)
$$

hence the composition

$$
\varphi: C \rightarrow C(Q) \rightarrow\left(C(Q)+J_{\mathrm{gr}}\left(C^{\prime}\right)\right) / J_{\mathrm{gr}}\left(C^{\prime}\right)=C^{\prime} / J_{\mathrm{gr}}\left(C^{\prime}\right)
$$

is a well-defined epimorphism of $\bar{G}$-graded $\bar{G}$-algebras. Consider the diagram

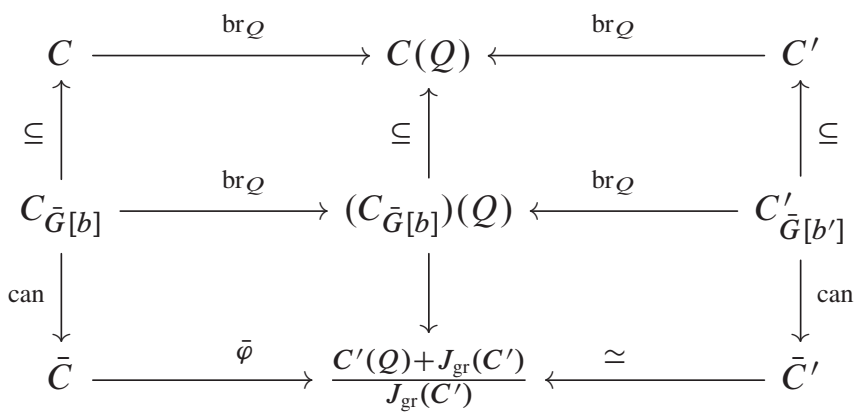

For any $\bar{x} \in \bar{G} \backslash \bar{G}[b]$, we have $\varphi\left(C_{\bar{x}}\right)=0$ because, otherwise, $C_{\bar{x}}$ would contain a homogeneous unit. If $\bar{x} \in \bar{G}[b]$, then $\varphi\left(C_{\bar{x}}\right)$ is the $\bar{x}$-component of $\bar{C}^{\prime}$. Furthermore, $\varphi\left(J\left(C_{1}\right)\right)=0$ since

$$
\left(C^{\prime} / J_{\mathrm{gr}}\left(C^{\prime}\right)\right)_{1}=C_{1}^{\prime} / J\left(C_{1}^{\prime}\right) \simeq b^{\prime} \mathcal{O} C_{H}(Q)^{N_{H}(Q)} / J\left(b^{\prime} \mathcal{O} C_{H}(Q)^{N_{H}(Q)}\right) .
$$

This proves that the kernel of $\varphi$ is $J_{\mathrm{gr}}(C)$; hence $\bar{\varphi}$ is an isomorphism, and then it also follows that $\bar{G}[b] \simeq \bar{G}\left[b^{\prime}\right]$. The commutativity of the remaining squares of the diagram is easily checked. 
The isomorphism $\bar{\varphi}$ induces a bijection between the blocks of $G$ covering $b$ and the blocks of $N_{G}(Q)$ covering $b^{\prime}$ by lifting idempotents in the above diagram via the canonical maps $C \rightarrow \bar{C}$ and $C^{\prime} \rightarrow \bar{C}^{\prime}$. The same bijection is obtained by lifting idempotents via the composition

$$
\varphi^{\prime}: C^{\prime} \rightarrow C(Q) \rightarrow\left(C^{\prime}(Q)+J_{\mathrm{gr}}\left(C^{\prime}\right)\right) / J_{\mathrm{gr}}\left(C^{\prime}\right) .
$$

By Lemma 3.4, this bijection coincides with the Brauer correspondence, so the theorem is proved.

The following statement immediately follows from 3.3 and Theorem 3.7.

Corollary 3.8. The composition of the Brauer map with the Fong-Reynolds equivalence induces an isomorphism $\bar{C} \simeq \bar{\ell}$ of $\bar{G}[b]$-graded $\bar{G}$-acted algebras.

\section{Morita invariance of the isomorphism $\bar{C} \simeq \bar{e}$}

4.1. Consider the $\bar{G}$-graded algebras $A=b \mathcal{O} G$ and $A^{\prime}=b^{\prime} \mathcal{O} G^{\prime}$ as in Section 2 . Assume that the $\left(A, A^{\prime}\right)$-bimodule $\tilde{M}$ and its $\mathcal{O}$-dual $\tilde{M}^{*}$ induce a $\bar{G}$-graded Morita equivalence between $A$ and $A^{\prime}$. By [14, Corollary 5.1.4], there is an isomorphism

$$
\Phi: C=C_{A}(B) \rightarrow C^{\prime}=C_{A^{\prime}}\left(B^{\prime}\right)
$$

of $\bar{G}$-graded $\bar{G}$-acted algebras, which is defined as follows. By the second Morita theorem [10, Theorem 12.12], there are $\bar{G}$-graded bimodule isomorphisms

$$
\tilde{\varphi}: \tilde{M}^{*} \otimes_{A} \tilde{M} \rightarrow A^{\prime}, \quad \tilde{\psi}: \tilde{M} \otimes_{A^{\prime}} \tilde{M}^{*} \rightarrow A
$$

such that

$$
\begin{aligned}
\tilde{\psi}\left(\tilde{m} \otimes \tilde{m}^{*}\right) \tilde{n} & =\tilde{m} \varphi\left(\tilde{m}^{*} \otimes \tilde{n}\right) & & \text { for all } \tilde{m}, \tilde{n} \in \tilde{M}, \tilde{m}^{*} \in \tilde{M}^{*}, \\
\tilde{\varphi}\left(\tilde{m}^{*} \otimes \tilde{m}\right) \tilde{n}^{*} & =\tilde{m}^{*} \psi\left(\tilde{m} \otimes \tilde{n}^{*}\right) & & \text { for all } \tilde{m}^{*}, \tilde{n}^{*} \in \tilde{M}^{*}, \tilde{m} \in \tilde{M} .
\end{aligned}
$$

Denote by $M, M^{*}, \varphi$ and $\psi$ the 1-components of $\tilde{M}, \tilde{M}^{*}, \tilde{\varphi}$ and $\tilde{\psi}$, respectively. Since $M$ and $M^{*}$ induce a Morita equivalence between $B$ and $B^{\prime}$, there are finite sets $I$ and $J$ and the elements $m_{j}^{*}, n_{i}^{*} \in M^{*}$ and $m_{j}, n_{i} \in M, i \in I, j \in J$ such that

$$
\tilde{\varphi}\left(\sum_{j \in J} m_{j}^{*} \otimes_{B} m_{j}\right)=1_{B^{\prime}}, \quad \tilde{\psi}\left(\sum_{i \in I} n_{i} \otimes_{B^{\prime}} n_{i}^{*}\right)=1_{B} .
$$

Then, for all $c \in C_{A}(B)$, we have that

$$
\Phi(c)=\tilde{\varphi}\left(\sum_{j \in J} m_{j}^{*} c \otimes_{B} m_{j}\right) .
$$


4.2. In particular, we may take an idempotent $i \in \gamma$ and the strongly $\bar{G}$-graded algebra $A_{\gamma}=i A i$ in the place of $A^{\prime}$. Since $A i A=A$, there is a finite set $J$ and elements $u_{j}, v_{j} \in A$ such that $\sum_{j \in J} u_{j} i v_{j}=1_{A}$. Then we get the isomorphism

$$
\Phi: C_{A}(B) \rightarrow C_{i A i}(i B i), \quad c \mapsto i c=c i,
$$

with inverse given by $c^{\prime} \mapsto \sum_{j \in J} u_{j} c^{\prime} v_{j}$, for all $c^{\prime} \in C_{i A i}(i B i)$.

This idea also applies in the case of the Morita equivalence between $A$ and $S \otimes A$, where $S=M_{r}(\mathcal{O})$ is a matrix algebra.

Theorem 4.3. With the notations of Section 2 , assume that there is a $\bar{G}$-graded basic Morita equivalence between $A=b \mathcal{O} G$ and $A^{\prime}=b^{\prime} \mathcal{O} G^{\prime}$. Denote $\bar{C}=\bar{C}_{A}(B)$, $\bar{C}^{\prime}=\bar{C}_{A^{\prime}}\left(B^{\prime}\right), \overline{\mathscr{C}}=\bar{C}_{\mathcal{A}}(\mathscr{B})$ and $\overline{\mathscr{C}}^{\prime}=\bar{C}_{\mathcal{A}^{\prime}}\left(\mathscr{B}^{\prime}\right)$. Then the diagram

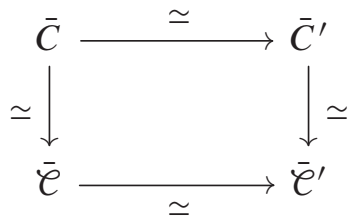

of $\bar{G}[b]$-graded $\bar{G}$-algebras is commutative, where the horizontal isomorphisms are induced by the $\bar{G}$-graded basic Morita equivalences given by the hypothesis and by 2.6, while the vertical isomorphisms come from Corollary 3.8.

Proof. Assume that the basic Morita equivalence is induced by the $\bar{G}$-graded $\left(A, A^{\prime}\right)$-bimodule $\tilde{M}$. Let $A_{\gamma}=i A i$ and $A_{\gamma^{\prime}}^{\prime}=i^{\prime} A^{\prime} i^{\prime}$. Then $i \tilde{M} i^{\prime}$ induces a $\bar{G}$ graded Morita equivalence between $A_{\gamma}$ and $A_{\gamma^{\prime}}^{\prime}$, which is also given by an embedding $f: A_{\gamma} \rightarrow S \otimes A_{\gamma^{\prime}}^{\prime}$ of $P$-interior $\bar{G}$-graded algebras, as in 2.5 .

We also use the $\bar{G}$-graded basic Morita equivalence between $\mathcal{A}=\mathcal{O} N_{G}\left(Q_{\delta}\right) b_{\delta}$ and $\mathcal{A}^{\prime}=\mathcal{O} N_{G^{\prime}}\left(Q_{\delta^{\prime}}\right) b_{\delta^{\prime}}^{\prime}$, as recalled in 2.6. Let $\mathcal{A}_{\lambda}$ be the source algebra of $\mathcal{A}$, and $\mathcal{A}_{\lambda^{\prime}}^{\prime}$ the source algebra of $\mathcal{A}^{\prime}$.

Now, consider the following diagram, in which all the maps are isomorphisms of $\bar{G}[b]$-graded $\bar{G}$-algebras.

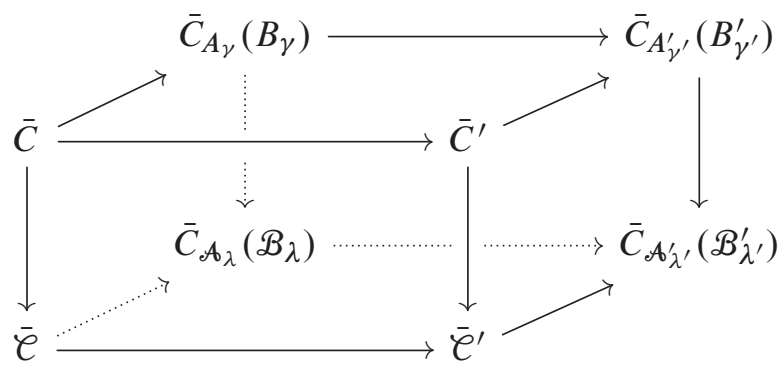


On the top face and the bottom face of the diagram, the maps come from the Morita equivalences $A \sim A^{\prime}, \mathcal{A} \sim \mathcal{A}^{\prime}$ and the embedding of the respective source algebras, as in 4.2 ; it follows immediately that the top face and the bottom face are commutative.

On the right face and the left face, the horizontal maps come from the embeddings of the source algebras as in 4.2 , while the vertical maps are induced by the Brauer map br $Q$, according to Theorem 3.7; hence the right face and the left face are commutative as well.

Recall that by [6, Section 3 and Remark 4.5.2], the extended Brauer quotient of $A_{\gamma}$ can be obtained by applying the usual Brauer construction to

$$
A_{\gamma} \otimes \mathcal{O}\left(N_{G}\left(P_{\gamma}\right) / C_{H}(P)\right) .
$$

By 4.2 and the above remarks, we deduce that the back face is commutative.

Consequently, the front face is also commutative.

We are mainly interested in the particular case when $G^{\prime}=N_{G}(Q)$ as in 3.1, in order to connect the above theorem with the Harris-Knörr correspondence.

Corollary 4.4. Let $G^{\prime}=N_{G}(Q), H^{\prime}=N_{H}(Q)$, and let $b^{\prime} \in Z\left(\mathcal{O} H^{\prime}\right)$ be the Brauer correspondent of $b$. Assume that $\tilde{M}$ induces a $\bar{G}$-graded basic Morita equivalences between $A$ and $A^{\prime}$. Then the bijection between the blocks of $A$ and the block of $A^{\prime}$ coincides with the Harris-Knörr correspondence.

Proof. Observe that our assumptions imply that $\mathcal{A}=\mathcal{A}^{\prime}=b_{\delta} \mathcal{O} N_{G}\left(Q_{\delta}\right)$. By Theorem 3.7, Corollary 3.8 and Theorem 4.3, we have the commutative diagram

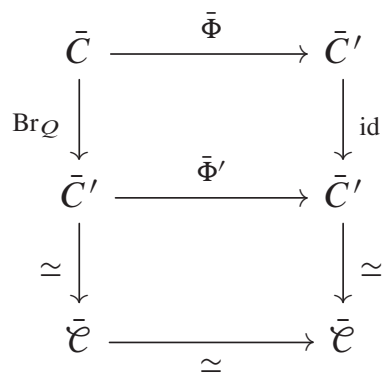

of isomorphisms of $\bar{G}[b]$-graded $\bar{G}$-acted algebras, where the maps $\bar{\Phi}$ and $\bar{\Phi}^{\prime}$ are induced by the given Morita equivalence. More precisely, $\bar{\Phi}^{\prime}$ is induced by the Fong-Reynolds equivalence between $A^{\prime}$ and $\mathcal{A}$, and a $\bar{G}$-graded basic Morita autoequivalence of $\mathcal{A}$. By [17, Lemma 4.5] (see also [4, Remark 9.6]), it follows that this auto-equivalence is the identity functor. Consequently, $\bar{\Phi}^{\prime}$ is the identity map of $\bar{C}^{\prime}$; hence the isomorphism $\bar{\Phi}$ is given by the Brauer map br $Q$. 


\section{Bibliography}

[1] M. Broué and L. Puig, Characters and local structure in G-algebras, J. Algebra 63 (1980), no. 2, 306-317.

[2] T. Coconeţ, A proof of a covering correspondence, Stud. Univ. Babeş-Bolyai Math. 58 (2013), no. 3, 289-295.

[3] T. Coconeț, $G$-algebras and Clifford extensions of points, Algebra Colloq. 21 (2014), no. $4,711-720$.

[4] T. Coconeț, A. Marcus and C.-C. Todea, Block extensions, local categories, and basic Morita equivalences, preprint (2018), https://arxiv .org/abs/1809.09323.

[5] T. Coconeț and A. Marcus, Group graded basic Morita equivalences, J. Algebra 489 (2017), 1-24.

[6] T. Coconeţ and A. Marcus, Remarks on the extended Brauer quotient, J. Algebra 491 (2017), 78-89.

[7] T. Coconeţ, A. Marcus and C.-C. Todea, Fusions and Clifford extensions, J. Group Theory 22 (2019), no. 1, 169-190.

[8] E. C. Dade, A Clifford theory for blocks, in: Representation Theory of Finite Groups and Related Topics, Proc. Sympos. Pure Math. 21, American Mathematical Society, Providence (1971), 33-36.

[9] E. C. Dade, Block extensions, Illinois J. Math. 17 (1973), 198-272.

[10] C. Faith, Algebra: Rings, Modules and Categories. I, Springer, New York, 1973.

[11] M.E. Harris and R. Knörr, Brauer correspondence for covering blocks of finite groups, Comm. Algebra 13 (1985), no. 5, 1213-1218.

[12] B. Külshammer and L. Puig, Extensions of nilpotent blocks, Invent. Math. 102 (1990), no. 1, 17-71.

[13] M. Linckelmann, The Block Theory of Finite Group Algebras, Cambridge University, Cambridge, 2018.

[14] A. Marcus, Representation Theory of Group Graded Algebras, Nova Science, Commack, 1999.

[15] A. Marcus, Twisted group algebras, normal subgroups and derived equivalences, Algebr. Represent. Theory 4 (2001), 25-54.

[16] L. Puig, On the Local Structure of Morita and Rickard Equivalences Between Brauer Blocks, Progr. Math. 178, Birkhäuser, Basel, 1999.

[17] L. Puig, Block source algebras in p-solvable groups, Michigan Math. J. 58 (2009), no. $1,323-338$.

[18] L. Puig and Y. Zhou, A local property of basic Morita equivalences, Math. Z. 256 (2007), no. 3, 551-562. 
[19] B. Späth, Inductive conditions for counting conjectures via character triples, in: Representation Theory-Current Trends and Perspectives, EMS Ser. Congr. Rep., European Mathematical Society, Zürich (2017), 665-680.

[20] B. Späth, Reduction theorems for some global-local conjectures, in: Local Representation Theory and Simple Groups, EMS Ser. Lect. Math., European Mathematical Society, Zürich, (2018), 23-61.

[21] A. Turull, Endoisomorphisms and character triple isomorphisms, J. Algebra 474 (2017), 466-504.

Received September 5, 2019; revised December 10, 2019.

\section{Author information}

Andrei Marcus, Faculty of Mathematics and Computer Science,

Babeş-Bolyai University, Cluj-Napoca, Romania.

E-mail: marcus@math .ubbcluj .ro 